\title{
A Numerical Investigation of Premixed Combustion \\ in Wave Rotors
}

M. R. Nalim

National Research Council.

D. E. Paxson

NASA Lewis Research Center, Cleveland, $\mathrm{OH} 44135$
Wave rotor cycles that utilize premixed combustion processes within the passages are examined numerically using a one-dimensional CFD-based simulation. Internalcombustion wave rotors are envisioned for use as pressure-gain combustors in gas turbine engines. The simulation methodology is described, including a presentation of the assumed governing equations for the flow and reaction in the channels, the numerical integration method used, and the modeling of external components such as recirculation ducts. A number of cycle simulations are then presented that illustrate both turbulent-deflagration and detonation modes of combustion. Estimates of performance and rotor wall temperatures for the various cycles are made, and the advantages and disadvantages of each are discussed.

\section{Introduction}

The wave rotor is a device that utilizes unsteady wave motion to exchange energy by direct work action between fluids, which may be chemically inert or reacting. It consists of a number of channels arranged about an axis; by rotation the ends of the channels are periodically ported to high and low-pressure ducts, which generate and utilize waves in the channels. Because the number of channels is large, the flow in the ducts is practically steady, and is directed to other steady flow components. An important feature is that as gases of a wide temperature range flow through the rotor, the mean channel-wall temperature is lower than the highest gas temperature. Rotational speed is low relative to turbomachines, and the geometry usually simpler, allowing greater strength and lower cost. For detailed descriptions of wave rotor principles and applications see Shreeve and Mathur (1985), Nalim (1994), and Welch et al. (1995).

A wave rotor acting as a pressure exchanger can be used (together with a conventional combustor) as a topping unit to enhance the performance of a gas turbine engine. Welch et al. (1995) have presented simulations based on validated codes, which indicate a substantial pressure gain possible between the compressor and the turbine. Similar pressure gain could also be obtained using an internal-combustion wave rotor. In this case, combustion occurs sequentially within the wave channels, each channel being periodically charged and discharged as it rotates past-properly-sized-and-timed inlet and outlet ports. Simplified combustion and wave processes are illustrated in the wave rotor sketch in Fig. 1. By accomplishing combustion on the rotor, the external combustor needed in a pressure-exchanger topping cycle is eliminated. So is the associated ducting, which might be long and unmanageably hot in some designs.

Combustion Modes. The implications of internal combustion and the feasible combustion modes are discussed in Nalim (1995). Rapid combustion is essential to minimize residence time and rotor size. The charge may be partially or fully premixed, and ignited by compression or other means. The feasible modes resemble combustion in various types of internal combustion (IC) engines. For relatively low inlet temperature (less than about $800 \mathrm{~K}$ for hydrocarbon fuel), premixing and sufficient turbulence are necessary to permit a high deflagration

Contributed by the International Gas Turbine Institute and presented at the 41 st International Gas Turbine and Aeroengine Congress and Exhibition. Birmingham. United Kingdom, June 10-13, 1996. Manuscript received at ASME Headquarter February 1996. Paper No. 96-GT-116. Associate Technical Editor: J. N. Shinn. flame speed when ignited by a "spark" of residual or injected hot gas. With higher inlet temperatures, a detonation mode becomes likely in premixed gas, or a nonpremixed, turbulent " diesel' 'combustion mode may be used. Only premixed combustion is considered here.

Combustion Simulation Goals. This work is a step toward simulation of internal-combustion wave rotors. Numerical modeling of combustion is a challenging research area. There is a strong interaction between the energy release by chemical reaction and the dynamics of fluid motion. Localized energy release creates steep gradients in temperature and other properties, which drive transport of species, momentum, and energy. Fluid turbulence, when present, may interact with the reaction to the extent of dominating its rate. A wide range of time scales and length scales are important for different phenomena.

In an internal-combustion wave rotor, the large-amplitude nonsteady motion typical of wave rotors combines with intermittent combustion. This imposes a heavy computational burden, especially for multidimensional calculations. In the case of shock-induced reaction and detonation, the numerical problems typically associated with shock resolution and ensuring accurate shock-speed are compounded by the chemical reaction. Because chemical induction time is a sensitive function of temperature, it is a challenge to obtain accurate chemistry near a shock when there is locally poor accuracy for energy and temperature. In the case of turbulence-enhanced deflagration, the numerical representation of turbulence, as well as the choice of a combustion model that appropriately combines chemical kinetic and turbulence effects, are both difficult issues. The computation should properly resolve the flame thickness and the multiple time scales and length scales (acoustic, diffusion, and reaction). Ideally, adaptive gridding is needed to efficiently compute the flame ptupagation.

The present work attempts only the simplest, one-dimensional, representation of combustion that is compatible with an existing wave-rotor design and simulation code (Paxson, 1995a; Paxson and Wilson, 1995). This uniform-grid, one-dimensional code has already allowed much progress in designing pressureexchange cycles, with rapid turnaround of computations on a single-processor workstation. It was desirable to create a similar tool for preliminary analysis and design of internal-combustion wave cycles. It is acknowledged that any one-dimensional representation of combustion processes will necessarily be rather crude, particularly when turbulence dominates. The intended approach is to select the model parameters to achieve simulation 


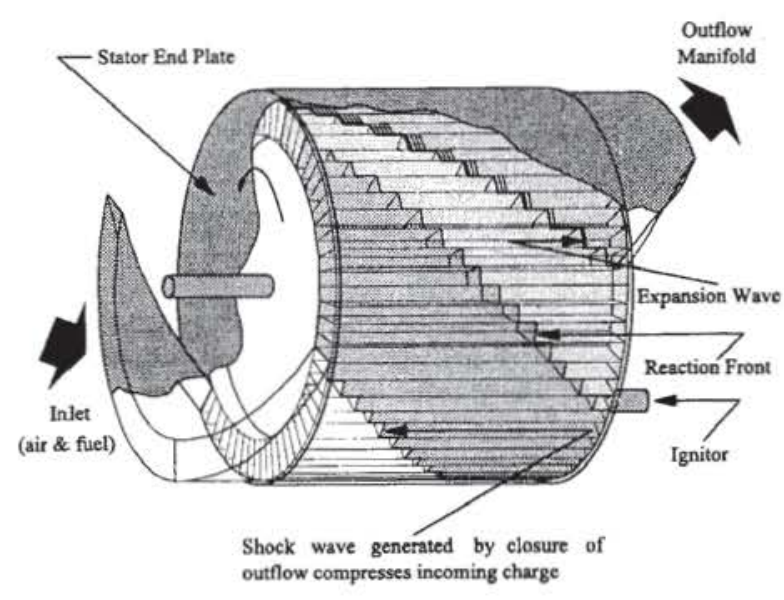

Fig. 1 Internal combustion wave rotor

of a desired combustion rate, and then estimate the required chemical and turbulence properties.

This paper is focused on the design of premixed-charge wave cycles, on understanding the flow dynamics relating the heat release to the pressure waves, and on estimating overall performance and material temperatures. The wave rotor model, governing equations for flow and combustion in the channels, and the numerical method used are described. A number of deflagration and detonation mode cycle simulations are then presented. The predicted pressure gains and wall temperatures are compared and the advantages and disadvantages of the various cycles are discussed.

\section{Wave Rotor Model}

The present model is based on a previous wave rotor simulation model for nonreacting gases (Paxson, 1992, 1995a). In this model, one-dimensional computation using a high-resolution CFD technique is performed for a single channel, neglecting interactions between channels. Losses due to the finite passage-opening time, leakage to the casing through the end gaps, heat transfer to the channel walls, and boundary layer viscous losses, are all treated by experimentally validated submodels (Paxson and Wilson, 1995). In addition to the CFD treatment of the flow in the channel, the cavities and the channel walls are treated by lumped-parameter models, and the ducts are modeled as steady, constant-area flows to obtain flow-homogenization losses. The overall pressure gain is calculated using averaged stagnation quantities computed from the absolute frame of reference, which takes the rotational speed into account.

Governing Equations for Channel Flow and Premixed Combustion. The present model assumes a calorically perfect gas, i.e., with a constant specific heat ratio $(\gamma)$. The composition of the charge at any time and location is described solely by a reaction progress variable $(z)$, which changes from 1 (pure reactant) to 0 (product) as combustion occurs, similar to Colella et al. (1986). Thus there will be one additional equation to be solved, besides the Euler equations of the nonreacting model. A simple representation of turbulence is included in the form of an eddy diffusivity.

The model numerically integrates the equations of motion in a single passage as it revolves past the ports and walls that comprise the ends of the wave rotor and establish the boundary conditions for the governing equations in the passage. Ports are specified by their circumferential location relative to some fixed point on the wave rotor casing, and by a representative pressure. temperature, and reactant fraction. With each time step the passage advances an angular distance specified by the angular velocity. If the flow is into the passage, the pressure and temperature are specified as stagnation values. If the flow is out of the passage, only the port pressure is required, and it is specified as a static value. Determination of the direction of the port flows at each time step is discussed in Paxson (1992).

The governing equations written in nondimensional form are:

$$
\frac{\partial \underline{w}}{\partial t}+\frac{\partial \underline{F}(\underline{w})}{\partial x}=\underline{S}(\underline{w})
$$

where vectors $\underline{w}$ and $F$ have the respective perfect-gas forms:

$$
\underline{w}=\left[\begin{array}{c}
\rho \\
\rho u \\
\frac{p}{\gamma(\gamma-1)}+\frac{\rho u^{2}}{2}+\rho z q_{0} \\
\rho z
\end{array}\right]
$$

$$
\underline{F}=\left[\begin{array}{c}
\rho u \\
\frac{p}{\gamma}+\rho u^{2} \\
u\left(\frac{p}{(\gamma-1)}+\frac{\rho u^{2}}{2}+\rho z q_{0}\right) \\
\rho u z
\end{array}\right]
$$

The nondimensionalization of pressure $(p)$, density $(\rho)$, and velocity $(u)$ has been obtained using a reference state $p^{*}, \rho^{*}$, and the corresponding sound speed $a^{*}$. The distance has been scaled by the passage length, $L$. The time has been scaled using the nominal wave transit time, $L / a^{*}$. The heat of reaction of the reactant gas, $q_{0}$, is assumed to be a constant. An alternative formulation is possible, in which the heat of reaction is treated like an external heat source, and the chemical energy term is not used. Although this simplifies the algebra and coding, and the computations were checked to be equivalent, the given formulation is more consistent with the use of conservation variables, and can be extended to treat multiple species and real chemistry with variable $q_{0}$.

The source vector, $S(w, x)$ includes contributions from the chemical reaction rate, turbulent eddy diffusion, and viscous forces and heat transfer at the walls. A leakage term is also added for the end gas. Without leakage, the source term is

$$
\underline{S}(\underline{w}, x)=\left[\begin{array}{c}
0 \\
\frac{\epsilon_{t}}{\operatorname{Re}^{*}} \frac{\partial^{2} u}{\partial x^{2}}+\sigma_{2} u|\rho u|^{0.75} \\
\frac{\epsilon_{t}}{\operatorname{Re}^{*}} \frac{\partial^{2}}{\partial x^{2}}\left(\frac{u^{2}}{2}+\frac{T}{(\gamma-1) \operatorname{Pr}_{t}}+\frac{z q_{0}}{\mathrm{Sc}_{t}}\right) \\
+\sigma_{3}|\rho u|^{0.75}\left(T-T_{\text {watl }}\right) \\
\frac{\epsilon_{t}}{\operatorname{Re}^{*} \operatorname{Sc}_{t}} \frac{\partial^{2} z}{\partial x^{2}}-\rho z\left(1-c_{1} z\right)\left\{\begin{array}{c}
K_{0} ; T \geq T_{0} \\
0 ; T<T_{0}
\end{array}\right\}
\end{array}\right]
$$

The forms of the wall source terms for viscous stress and heat transfer and their coefficients $\sigma_{2}$ and $\sigma_{3}$ are based on semiempirical correlations. The expressions and definitions for the combustion rate and eddy diffusion terms are discussed below.

Combustion Rate. In general, the rate of combustion at a given location in the flow will depend on the local composition, temperature, pressure, and turbulence properties. The mechanisms of combustion are quite different for turbulent deflagration and for detonation of premixed charges, and different forms of the rate equation are expected. In each case we represent combustion by a finite-rate, single-step reaction. 
For the calculation of shock-ignited reaction and detonation, the rate $(R)$ is assumed to be proportional to the reactant fraction, and to have an Arrhenius-type dependence on temperature. The rate coefficient is based on available single-step reaction kinetic models. Usually, a large activation energy is assumed, and ignition temperature kinetics are used, i.e., the rate coefficient is zero below a threshold (ignition) temperature $\left(T_{0}\right)$, and is a constant $\left(K_{0}\right)$ above it. This mode is activated by setting $c_{1}=0$ in Eq. (4).

For the calculation of turbulent deflagration, the turbulence model described in the next subsection is activated. Here also, ignition-temperature kinetics are used, but the rate is assumed to be proportional to both the reactant and the product fractions, i.e., $R \propto z(1-z)$ by setting $c_{1}=1$ in the source vector, based on the suggestion of Magnussen and Hjertager (1976). This implies that the reaction can occur only at a propagating flame surface. The rate coefficient must be assigned phenomenologically, based on an estimate for the reaction timescale, which may be influenced by both chemistry and turbulence.

No special model is used for the ignition process to initiate a deflagration. In the cases considered here, initiation takes place by recirculation of hot combustion gas from leading channels, and by residual hot gas in the channel. Cavity leakage was also observed to initiate a flame in some simulations not discussed here. The one-dimensional treatment does not capture the penetration and vortex mixing effect of a jet of hot gas injected through an orifice smaller than the channel width.

Turbulence Model. The effect of turbulence is approximated by the use of an eddy diffusivity, which results in diffusive fluxes of $z$, energy, and momentum, proportional to their respective streamwise gradients. In Eq. (4), $\mathrm{Re}^{*}$ is a Reynolds number based on the reference state $\rho^{*}, a^{*}$, and $L ; \epsilon_{f}$ is the eddy viscosity scaled by the molecular viscosity. The formulation permits the use of different diffusivities for mass, momentum, and heat, by specifying the turbulent Prandtl number $\mathrm{Pr}_{\text {, }}$ and turbulent Schmidt number $\mathrm{Sc}_{\imath}$.

Clearly, such a model has little predictive value, because the role of turbulence in flame propagation is much more complex than simply eddy diffusion. Within the constraints of a onedimensional calculation, however, there is not much scope for worthwhile sophistication. It is comforting that the flame propagation rates calculated showed an appropriate sensitivity to the values for the model parameters, as discussed later. A more detailed model and multidimensional computations are needed to examine the real physics of turbulent flame propagation.

System Model and Wall Temperature Calculation. The system layout for wave rotor simulation is shown schematically in Fig. 2. Boundary conditions for the end or port regions of the channel flow are generally supplied as stagnation states. These are either provided directly by the user, as in the case of the port leading from the upstream compressor, or calculated by lumped-capacitance models of the rotor housing space and the recirculation passages. The space between the rotor and the housing, to and from which leakage occurs, is lumped as a single cavity. The pressure differences between the cavity and the channels, together with the specified gap between rotor and endwalls, govern the leakage flow via a source term in the first and last computational cells of each channel. The recirculation ducts are also lumped together as if they were a single cavity. A stagnation pressure loss proportional to the square of the mass flow is imposed on the flow going through the recirculation loop. The downstream turbine could also be modeled as a cavity and valve; however, in this study, the exhaust port pressure was held constant based on previous calculations for topping cycles (Paxson, 1995b). In this paper, the term lumped-capacitance implies that the kinetic energy of the flows in the components is neglected. Thus, they may be modeled using only mass and energy conservation equations.

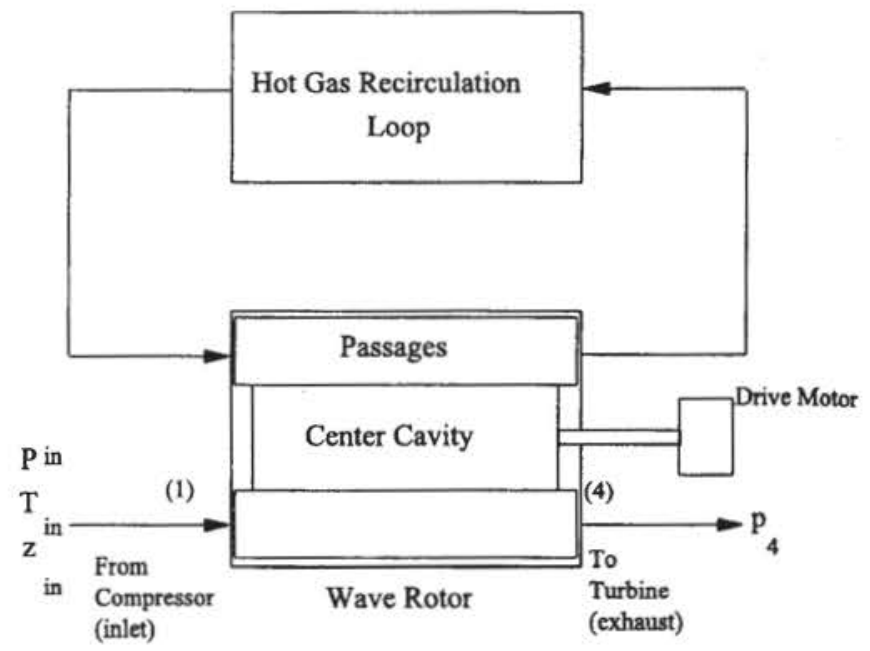

Fig. 2 System layout

The stagnation boundary conditions supplied by the user or component models are used by each channel in the CFD code to determine the state of the so-called image cell at the next instant of time. The code is capable of assessing whether a given condition will lead to inflow or outflow in a given channel. This allows robust operation of the simulation even in some off-design conditions where a portion of the flow in a given port may be into the rotor and a portion out of the rotor. For outflow conditions, only the boundary pressure is used, and it is treated as a static value. For inflow conditions some accounting is made if the flow in the duct is not aligned with the passage (i.e., shaft work into the system). For both inflow and outflow conditions accounting is made at the boundaries for the effects on the flow of those channels, which are only partially opened to a port: so-called finite opening effects. The ducts leading to and from the ports are assumed loss free (isentropic); however, a constant area mixing calculation is used in outflow ports to account for losses due to non-uniformities in the flow.

A lumped capacitance method is also used to track the wall temperatures, as described by Paxson (1995a), except that the channel side walls also contribute to the heat transfer, and are assumed to be at the same temperature as the upper and lower walls. Longitudinal conduction in the rotor is not allowed in order to obtain the worst case wall temperatures. Thus, each slice of the rotor that is in contact with a channel computational cell is treated as a separate lump. The computed steady-state wall temperature may be thought of as a time-averaged gas temperature, but weighted for heat transfer.

\section{Numerical Method}

Equation (1) is integrated numerically as follows:

$$
\underline{w}_{i}^{n+1}=\underline{w}_{i}^{n}-\left(\underline{f}_{i+1 / 2}^{n}-\underline{f}_{i-1 / 2}^{n}\right) \frac{\Delta t}{\Delta x}+\underline{s}_{i}^{n} \Delta t
$$

where the numerical flux estimate is

$$
\begin{aligned}
\underline{f}_{i+1 / 2}^{n}=\frac{\underline{F}_{i+1}^{n}+\underline{F}_{i}^{n}}{2}-\frac{1}{2} \underline{\phi}_{i+1 / 2}^{\mathrm{Roc}} & \\
& +\frac{\Delta t}{4}\left([A]_{i+1}^{n} \underline{S}_{i+1}^{n}+[A]_{i}^{n} \underline{S}_{i}^{n}\right)
\end{aligned}
$$


and the numerical source term is

$$
\underline{s}_{i}^{n}=\frac{1}{2}\left(3 \underline{S}_{i}^{n}-\underline{S}_{i}^{n-1}\right)
$$

The term $\phi^{\text {Roc }}$ in Eq. (5) refers to the flux-limited dissipation based on the approximate Riemann solver of Roe (1986) for Eq. (1) without a source vector. The matrix $[A]$ is the Jacobian of the flux vector $F$. The superscript $n$ and subscript $i$ are indexes for the discrete temporal and spatial steps, respectively. This scheme has the advantage of being formally second-order accurate in time and space when the flow is smooth yet maintaining the high resolution of Roe's method in the vicinity of shock waves. Furthermore, as the source strength approaches zero, the scheme becomes monotonic, which is physically correct.

There are additional requirements on the numerical scheme to preserve the physical meaning of $z$, which are not inherent in the governing equations: $z$ should remain in the range from 0 to 1 , and the combustion source term for the rate of change of $z$ in Eq. (4) should be negative or zero. These are usually satisfied by the use of physically meaningful initial and boundary conditions, well-behaved source term discretization, and a stable numerical scheme with monotonic source-free behavior. A simple first-order stiffness scheme was created for the combustion source term, by dividing it by a factor $\left(1+K_{0} \Delta t\right)$, to ensure positivity of $z$. In practice, this was found unnecessary because the time step for stability of the Riemann solution was always much smaller than the reaction time scale. There are situations, unrelated to the source term, in which numerical integration of the Riemann problem results in slightly out-ofrange $z$, even with a monotonic scheme (Larrouturou, 1991); however, in the simulations to be presented, they did not arise.

Since the time constants associated with transients in the wall temperatures and cavity properties are much larger than a complete wave cycle, these quantities are treated as constants for each wave cycle, and then updated using simple Euler integration (Paxson, 1995a). The actual rotor thermal inertia and cavity volumes do not affect a steady-state solution. Hence, the smallest values that allow stable computation are used for rapid convergence to a periodic, zero-net-flux solution, and steadystate wall, cavity, and duct properties.

Test Cases and Grid Independence. A number of reaction-wave test problems were solved to ensure that the numerical scheme was stable and produced meaningful solutions. These included the development of detonations in various frames of reference. For a direct test of accuracy in computing detonation speed, the boundary and initial conditions were set up to match a steady Chapman-Jouguet $(\mathrm{C}-\mathrm{J})$ detonation in a perfect gas with $\gamma=1.2$, and fixed heat release, $q_{0}=30$, where the reference state is that of the unburned gas. After a brief transient, due to the fact that the prescribed initial step profile neglects the thickness of the reaction zone, the detonation becomes steady with the propagation speed correctly matching the prescribed inflow speed. The classical Zeldovich-von Neumann-Doring (ZND) structure appears, with the computed von Neumann pressure spike within 3 percent of the theoretical value of 30.9 (with $\Delta x=0.005, K_{0}=30.0, T_{0}=2.0 T_{1}$ ). While the detonation speed was independent of reaction rate parameters and grid spacing, the pressure spike was underpredicted for coarser grids or lower $T_{0}$. Computations of overdriven and underdriven detonations were also qualitatively correct.

It is not equally straightforward to directly compare turbulent flame computations with any data or with realistic models, as the one-dimensional model rate parameters have limited physical meaning. In a sense, however, the inverse of $K_{0}$ can be related to the dominant reaction time scale, which is expected to be the turbulence time scale. It has been found experimentally (Heywood) that highly turbulent premixed flames, such as those found in IC engines, have flame speed $\left(s_{t}\right)$ comparable to the turbulence intensity, $u^{\prime}$, which should imply

$$
s_{t} \propto u^{\prime} \propto \sqrt{\frac{K_{0} \epsilon_{l}}{\operatorname{Re}^{*}}}
$$

In computations of flame propagation in a closed tube, this relationship was seen to be roughly preserved with a proportionality constant in the range of 1.0 to 1.2 , for values of $\epsilon_{t}$ from 250 to 1000 , and $K_{0}$ from 8 to 40 , all other variables being fixed at typical values $\left(\mathrm{Re}^{*}=8.3 \times 10^{6}, \mathrm{Pr}_{t}=\mathrm{Sc}_{t}=1.0, q_{0}\right.$ $\left.=4.0, T_{0}=1.5, \gamma=1.33\right)$. The flame speeds computed are also sensitive to changes in $q_{0}$ and $T_{0}$. For this same range of variables, grid-independent solutions were achieved for values of $\Delta x$ less than 0.005 , i.e., 200 cells in a channel length. Coarser grids resulted in exaggerated, grid-dependent flame speed.

\section{Deflagration Mode Wave Cycles}

Several possible wave cycles using turbulent deflagration were simulated. Because it is likely that low-pressure-ratio engines will use this mode (Nalim, 1995), the simulations assumed a design similar to a throughflow pressure-exchange wave rotor optimized for a small engine with an upstream compressor pressure ratio of approximately 8 (Paxson, 1995b). The major design parameters for such a reference wave rotor, listed in Table 1, are retained in nondimensional form except as noted. The reference state for nondimensionalization of variables is the stagnation state of the inlet to the wave rotor. In all the simulations, $\mathrm{Re}^{*}=8.3 \times 10^{6}, \mathrm{Pr}_{t}=\mathrm{Sc}_{t}=1.0, \gamma=1.353$. The simulations are presented as space-time contour diagrams of gas density and reactant fraction, over a full rotor revolution, with positive time in the upward vertical direction. The port timings are indicated by the breaks in the side borders representing the end plates. It is noted that the gas dynamics of a pressuregain wave rotor allows, in each cycle, only partial discharge of the combustion gas to the higher-pressure exhaust port, while fresh charge enters from the lower-pressure inlet port.

Fast-Burn Reverse-Flow Cycle. In this mode, the wave rotor is designed for opposed pairs of reverse-flow cycles, with the one inflow and one outflow port at each end of the rotor, as illustrated in the computed wave diagram of Fig. 3. With exact symmetry of the port placement and the resulting gas dynamics, there will be a resident layer of gas, which moves from side to side but does not leave the channel.

Each wave cycle is required to be completed in about the same half-revolution time as the corresponding pressure-exchange cycle. This requires very fast combustion, and almost instant ignition. The cycle is designed to provide hot gas recirculation from leading channels, via a transfer passage, to create a torch jet into the premixed charge. It is assumed that the hot residual gas, heated by combustion and repeated compression, also initiates a flame in the charge. The illustrated simulation was obtained by setting $K_{0}=28.0, T_{0}=1.5$, and $\epsilon_{t}=1000.0$. The inlet mixture is uniform, and $q_{0}$ is 3.42 to provide an overall temperature ratio of 2.2. Based on Eq. (8), and examination of the simulation, the corresponding flame speed is estimated to be $25 \mathrm{~m} / \mathrm{s}$ for the candidate engine operating at standard ambient

Table 1 Reference wave rotor dimensions and design performance

\begin{tabular}{lll}
\hline \hline Mean Rotor Radius & $8.15 \mathrm{~cm}$. & $(3.2 \mathrm{in})$. \\
Rotor Length & $15.24 \mathrm{~cm}$ & $(6.0 \mathrm{in})$. \\
Rotor Passage Height & $2.18 \mathrm{~cm}$. & $(0.86 \mathrm{in})$. \\
Rotational Speed & $16800 \mathrm{rpm}$ & \\
Cycles/Revolution & 2 & \\
Number of Passages & 52 & \\
Mass Flow Rate & $\approx 2.3 \mathrm{~kg} / \mathrm{s}$ & $(5.0 \mathrm{lbm} / \mathrm{s})$ \\
$\mathrm{P}_{4} / \mathrm{P}_{1}$ (Fig. 2) & 1.23 & \\
$\mathrm{~T}_{4} / \mathrm{T}_{1}$ & 2.21 & \\
\hline \hline
\end{tabular}



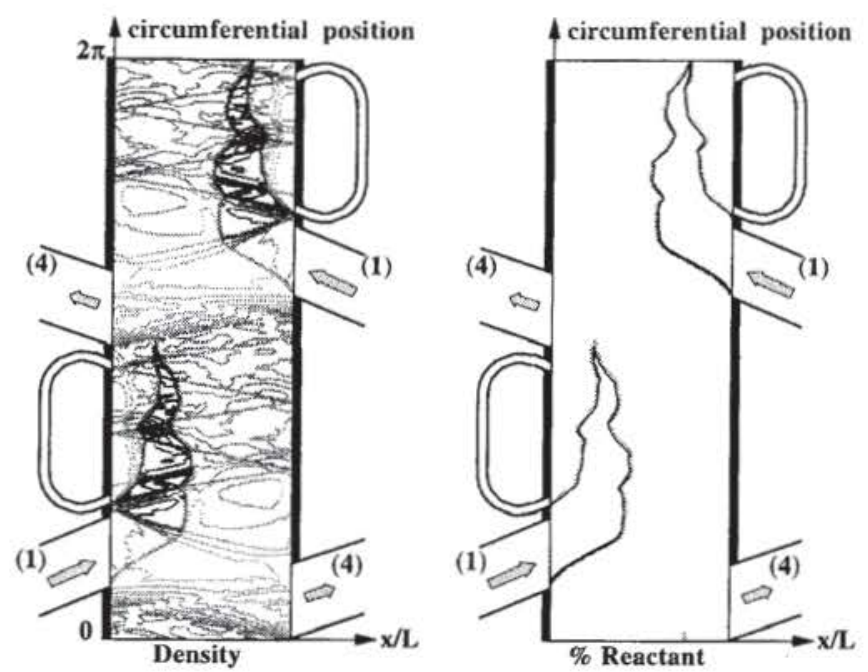

Fig. 3 Fast-burn reverse-flow cycle

temperature. This is at the high end of common IC engine experience, where $10 \mathrm{~m} / \mathrm{s}$ might be more typical.

A fast-burn throughflow cycle, which completes combustion of the charge in each half-revolution, would be very similar to this reverse-flow cycle in flame pattern and performance.

Slow-Burn Throughflow Single Cycle. This design is intended for relatively slow-burning mixtures and conditions. It has only one cycle per revolution, with the inlet and exhaust ports on opposite ends. In the simulation illustrated in Fig. 4, the inlet charge is stratified so that the middle one-fifth of the air has no fuel $(z=0)$. Ignition is similar to the last case. Here, the flames have about thrice the time to complete combustion, and a fifth less distance to travel. Flame temperatures are higher, with $q_{0}=4.275$, to retain the overall temperature ratio of 2.2 . The simulation shown used $K_{0}=6.0, T_{0}=1.5$, and $\epsilon_{t}=500.0$, consistent with a flame speed about one-third that of the fastburn simulation, based on Eq. (8). Combustion is completed before discharge and, at this level of diffusivity, some temperature stratification persists in the exhaust.

The mass flow rate in this wave rotor will be half that of the reference design, or conversely, a given flow rate will dictate double the rotor size. This may be a crippling penalty, and a solution to the problem is presented in the next case.
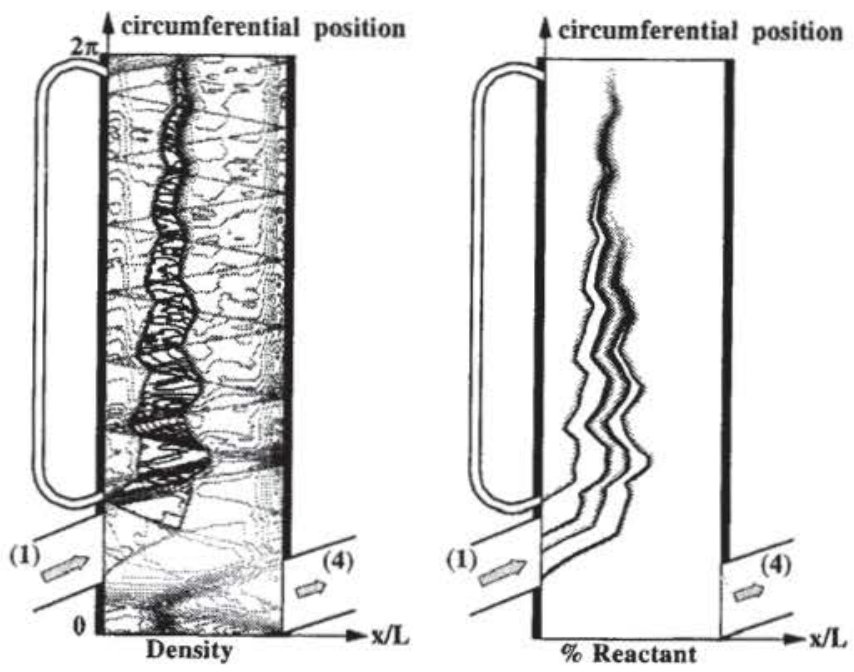

Fig. 4 Slow-burn throughflow single cycle
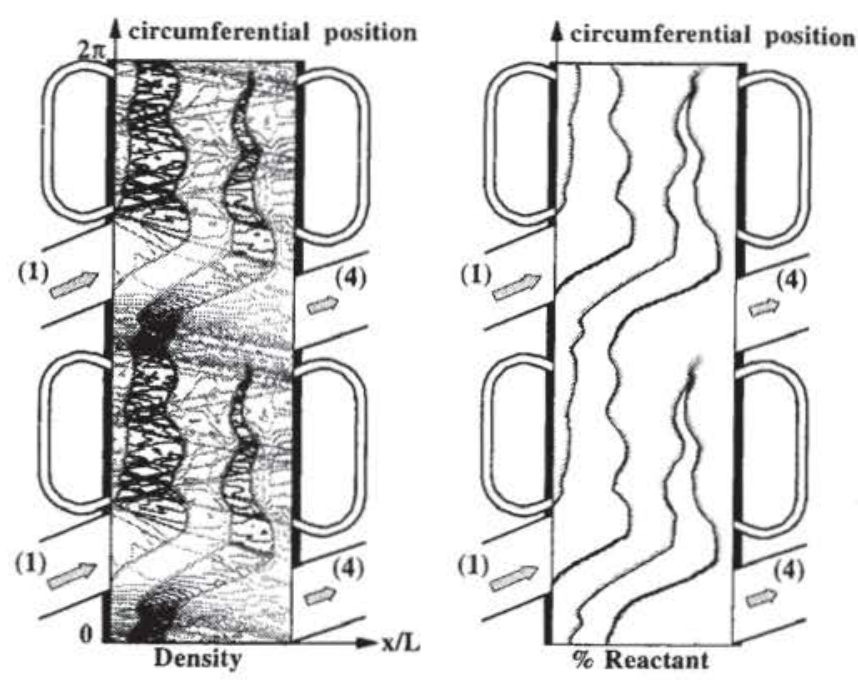

Fig. 5 Slow-burn throughflow dual cycle

Slow-Burn Throughflow Dual Cycle. If combustion is very slow, combustion of a charge introduced in one cycle may not be completed before the next discharge process. In a twoport pressure-gain cycle, optimized for an overall temperature ratio of 2.2 , only about 60 percent of the gas in the channel is discharged in each cycle. Therefore, a dual throughflow cycle may be envisioned, in which the fresh charge introduced at one end of the channel is burned over a period of two cycles, such that all the gas discharged at the other end has completely burned before final expansion. The throughput mass flow rate of the reference design is now recovered. The corresponding simulation, shown in Fig. 5, used $K_{0}=10.0, T_{0}=1.5$, and $\epsilon_{t}$ $=500.0$. Equation (8) implies flame speed about two-fifths that of the fast-burn case, which is roughly consistent with the relative combustion durations of the two cases, if it is noted that combustion is slowed during the low-pressure period. In this case, recirculation loops are provided at both ends to ensure quick ignition and complete combustion.

\section{Detonation Mode Wave Cycles}

The detonation mode is likely to be used for high-pressureratio engines with inlet temperature close to the autoignition temperature for the fuel used. For the purpose of this paper, however, most of the design parameters of the reference smallengine wave-rotor (Table 1) are retained in nondimensional form. We also keep $\mathrm{Re}^{*}=8.3 \times 10^{6}, \mathrm{Pr}_{t}=\mathrm{Sc}_{t}=1.0, \gamma=$ 1.353. The throughput mass flow rate is approximately doubled relative to the reference design by doubling the number of cycles per revolution (to four) to take advantage of the rapidity of detonative combustion. The rotor speed is adjusted slightly to match the strong combustion-driven waves. The contour diagrams in this section cover only half a revolution, and temperature is shown instead of density because detonation involves unly a slight change (increase) in density.

Throughfiow Cycle. The throughflow cycle presented in Fig. 6 has a stratified inlet charge, with no fuel in the first onefifth of the port duration to avoid the possibility of flashback or premature ignition of the nearly detonable charge. This buffers the fuel from the residual hot gas in the channel. To compensate and maintain the overall temperature ratio at about 2.2 , the simulation uses $q_{0}=3.785$. The reaction rate used is $K_{0}=$ 10.0 , with $T_{0}=1.2$ and $\epsilon_{s}=100.0$. At this low level of diffusivity, the temperature stratification due to the buffer layer persists in the exhaust.

The detonation is initiated by coalescing compression waves generated by closing the exhaust port while there is still signifi- 
cant flow velocity. The inlet port is closed just before the detonation wave arrives, and the exhaust port is designed to open at the time that the reflected detonation-generated wave reaches the opposite end, based on preliminary computations. This requires the rotor speed to be about 6 percent slower than the reference design, with four cycles per revolution. When a converged solution was obtained, however, it was found that the detonation wave timing had changed somewhat.

Reverse-Flow Cycle. The reverse-flow cycle of Fig. 7 also has a stratified inlet charge, but with fuel concentrated in the middle three-fifths of the port. While the leading buffer layer prevents pre-ignition as before, the trailing buffer layer is used to attempt to ensure that combustion is completed within the channel, and no unburned fuel reaches the exhaust port. This allows a finite land between the inlet and exhaust ports without weakening the detonation. The ideal timing is to open the exhaust immediately when the detonation-generated wave reaches the end wall, and the rotor speed in the simulation is adjusted to be about 12 percent faster than the reference design, to match the estimated timing. In practice, the time of initiation of the detonation varies from cycle to cycle, as seen in Fig. 7. Frequently, the detonation is late, and runs into the exhaust expansion, resulting in performance loss, or incomplete combustion, despite the buffer zone.

Another drawback of the reverse-flow cycle is that the residual gas, which never leaves the channel, becomes very hot due to repeated traversal by strong shock waves, resulting in increasing expansion of this gas and reduced room for fresh charge. In practice, the temperature of this gas will be limited by loss of heat to the walls (if cooled) and axial diffusion in the gas. It was found that convergence of this solution to the approximate steady state of Fig. 7 required an eddy diffusivity of $\epsilon_{t}=1000.0$, with $K_{0}=20.0, T_{0}=1.2, q_{0}=5.0$.

Precise and repeatable timing of detonation initiation is difficult to ensure in any detonation mode. The sensitivity to charge conditions may be exaggerated because of the ignition-tempera-
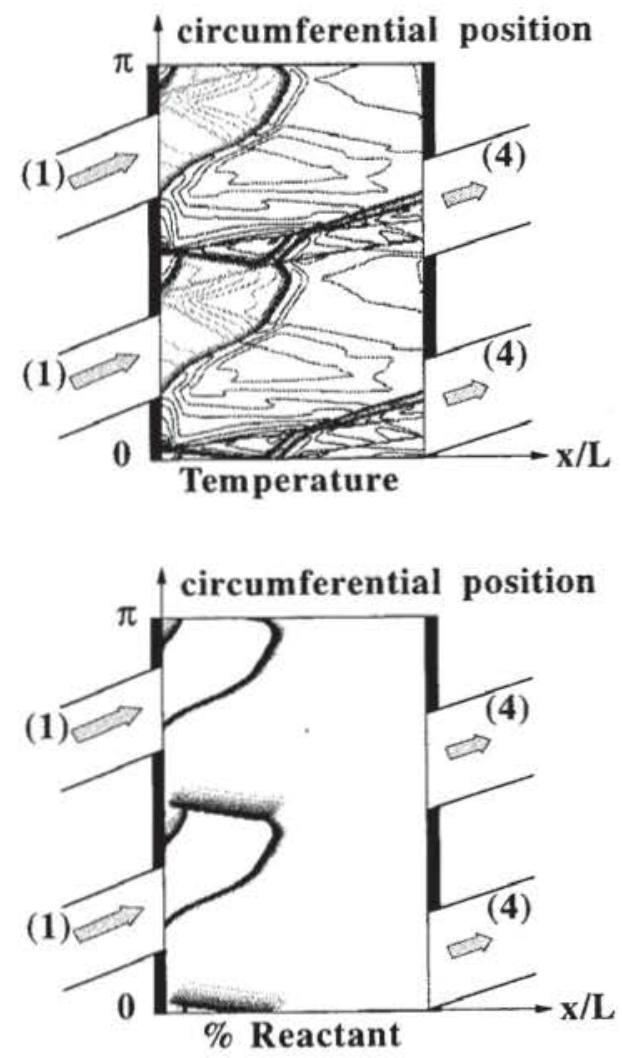

Fig. 6 Throughflow detonation-mode cycle ture kinetics used in the simulations, but will exist even with more realistic chemistry. Delayed initiation is less of a concern for the throughflow cycle, where there is adequate time for fuel burn-up. If the exhaust opening is timed without regard for the detonation-generated wave, a poor exhaust velocity profile and strong unsteadiness or nonuniformities in the outflow may result. Delayed exhaust opening, timed for later reflections of the wave, would throw away the fast-burn advantage of detonation, and increase the wall heat load and leakage.

\section{Comparison of Performance and Wall Temperatures}

For all three cases of deflagration cycles, the pressure gain (averaged exhaust flow pressure over inlet pressure) was 1.20 \pm 0.05 . The slow-burn single cycle has a leakage coefficient set to half that of the other two to compensate for the increased time at high pressure, since it is not intended to examine the absolute effect of leakage here. It seems remarkable that the slow-burn dual cycle suffers very little from the fact that some combustion may occur at low pressure, when it is thermodynamically unfavorable for pressure gain. In the combustion model used, the rate of reaction is slowed during that time, as would be expected in reality. The pressure gain computed for the throughflow detonation cycle is 1.22 . Pressure gain for the reverse flow detonation cycle is not reported because an acceptably steady solution with well-timed and complete combustion was not achieved.

For comparison, the equivalent throughflow pressure-exchanger wave rotor of Paxson (1995b) has a computed pressure gain of 1.23 . It should be noted that no attempts were made to optimize any of the cycles for geometry, rotor speed, wave timings relative to port timings (except detonation, without success), correctness of duct angles for the velocity profiles, etc. The port durations for the hot gas recirculation loops are made greater than the channel width, due to the present limitation of the code for opening/closing calculations. This results in excessive recirculation flow (sometimes 20 percent of net flow), which may affect performance adversely. The waves generated by these ports also appear sometimes to cause strong nonuniformity in the exhaust. With good optimization of the cycles, improved performance is expected.

The steady-state wall temperature profiles of the simulations differ significantly, as shown in Figs. 8 and 9. Recall that longitudinal conduction in the wall is absent in these computations. The mean wall temperature is indicated by a horizontal bar for each case. This is an indicator of the rotor temperature when its conductivity is high. It may differ slightly from the temperature computed if a uniform wall temperature was assumed (infinite conductivity), since local heat transfer depends on the local temperature difference.

Converting the deflagration mode curves of Fig. 8 to absolute temperatures, assuming a turbine inlet temperature (TIT) of $1300 \mathrm{~K}$, the peak wall temperature for the throughflow dual cycle is roughly equal to this TIT. For the reverse flow and throughflow single cycles, the peak wall temperatures are respectively about $110 \mathrm{~K}$ and $190 \mathrm{~K}$ higher than the TIT. The mean wall temperature for the throughflow dual cycle is $250 \mathrm{~K}$ lower than TIT, whereas the mean temperature for the other two cycles are about equal, both $70 \mathrm{~K}$ below TIT.

For the detonative cycles (Fig. 9), conversion of temperature to absolute values based on same TIT and temperature ratio assumes a detonable fuel-air mixture at low inlet temperature. The throughflow cycle has a peak wall temperature close to TIT and a mean temperature $170 \mathrm{~K}$ below TIT. This is much cooler than the throughflow deflagrative single cycle, due to the relatively short time at high temperature, and the passage of the cold buffer layer. There are two curves for the reverse-flow cycle, one for a simulation with $\epsilon_{t}=1000.0$ and the other with $\epsilon_{1}=100.0$. These are intended only for a relative comparison, since the solutions were not sufficiently well converged for 

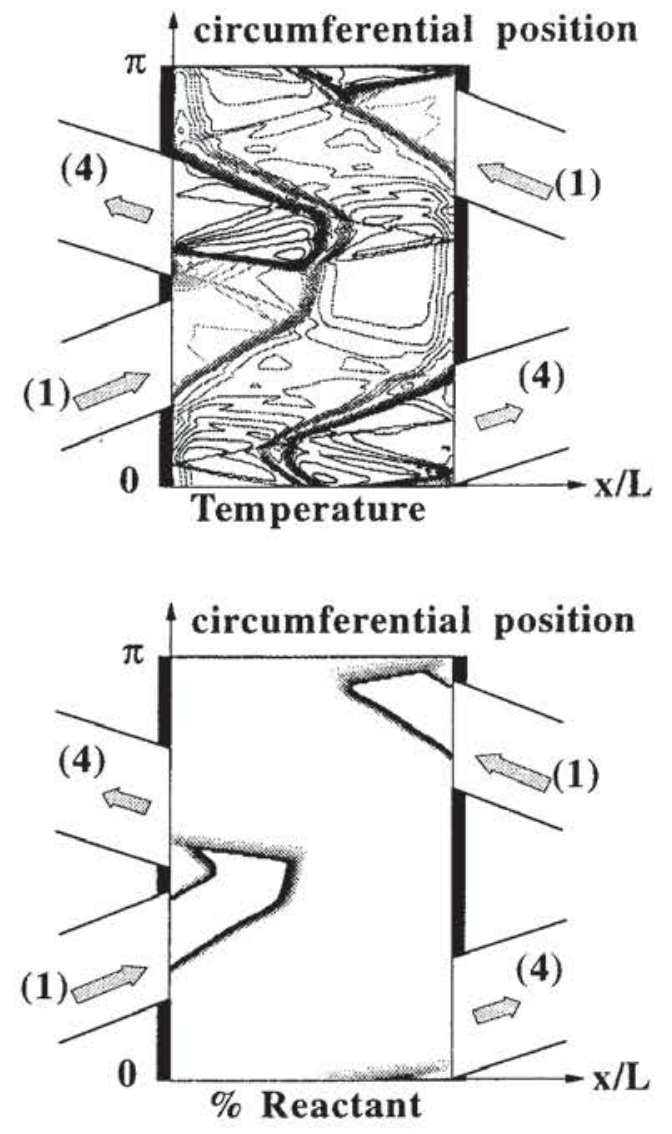

Fig. 7 Reverse-flow detonation-mode cycle

quantitative comparison. It is also noted that the heat transfer model validated for flow in channels may not be as accurate for flows involving detonations, which have structure and transverse oscillations that may substantially enhance heat transfer.

\section{Concluding Remarks}

A computational model for flow and premixed combustion in wave rotors has been developed that allows rapid simulation of deflagrative and detonative combustion mode wave cycles. The one-dimensional treatment of channel flow and combustion is coupled with validated models for loss mechanisms and external component interaction effects to provide realistic estimates of wave rotor performance and temperatures. The example simulations demonstrate that useful wave rotor cycles can be designed that can utilize a great range of combustion rates, depending on the fuel chemistry and turbulence properties pro-

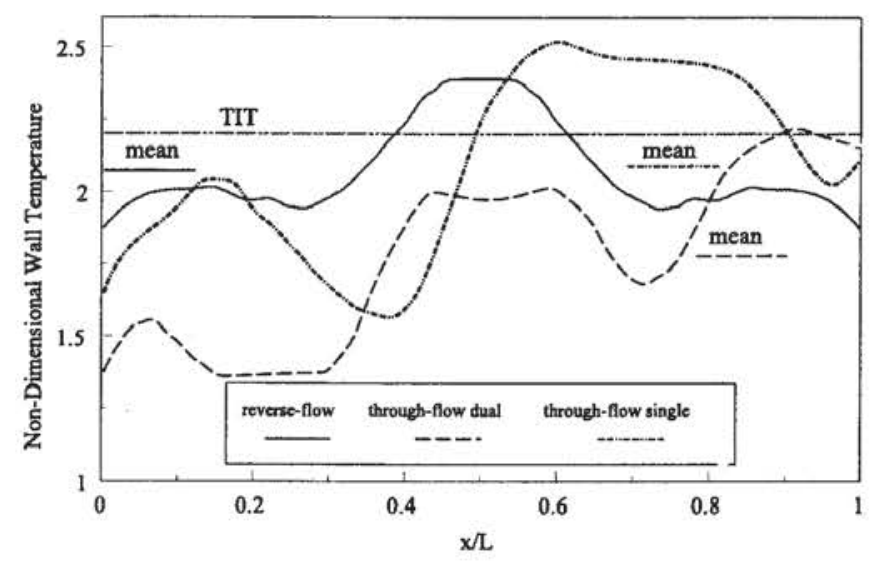

Fig. 8 Deflagration mode wall temperatures

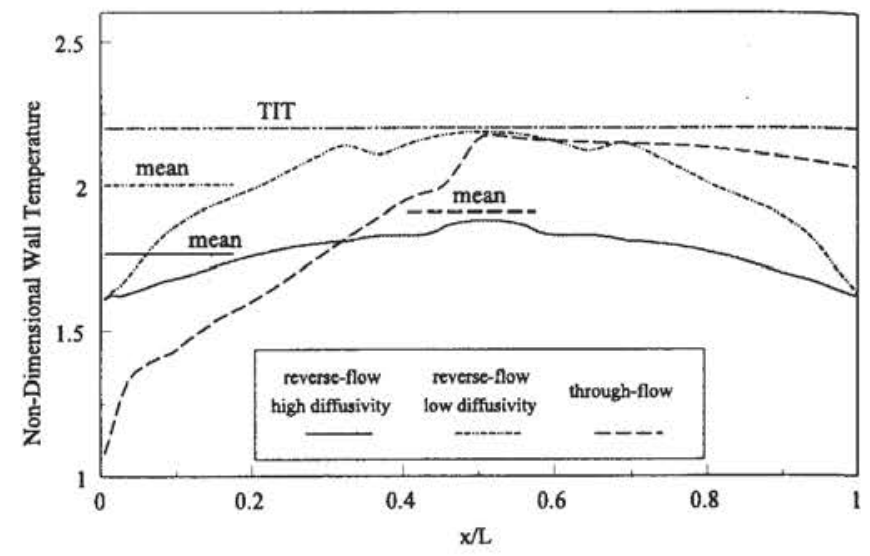

Fig. 9 Detonation mode wall temperatures

vided. The highest throughput mass flow rates for a given rotor size can be achieved with detonative mode cycles. However, the sensitivity of this mode to port timing and boundary conditions is a significant issue. The throughflow detonative cycle is relatively well-behaved.

This exercise calls attention to the possibility that combustion within a wave rotor may have an inherent potential for instability, as the wave dynamics and combustion rates become closely coupled. Because combustion is naturally autocatalytic, perturbations that reduce the combustion rates may rapidly degenerate to a flame-out state. This may be the most significant drawback relative to an external-combustion pressure-exchange wave rotor. The use of hot gas recirculation for ignition of deflagration would seem to only add to the dependence on positive feedback. Nevertheless, the deflagration simulations appeared to be quite stable compared to the autoignited detonations. Further exploration of the sensitivity and stability issues for different combustion rate formulations and parameters is necessary.

Very high flame speeds will be needed for reverse- or throughflow deflagration mode cycles, which attempt to provide the same throughput mass flow rates as an equivalent equalsize pressure exchanger using an external combustor. Instead, it is possible to design a throughflow cycle that accommodates relatively slow flames, simply by allowing combustion of the charge to continue for the duration of two cycles. Since pressure-gain wave-rotor cycles always have incomplete purging, there is the opportunity for each particle of charge to complete combustion before expansion to exhaust. Injection of recirculated hot gas can be provided at both ends to ensure early ignition and complete combustion. In effect, this results in four flame sites during the confined pressure-rise combustion periods, while the naturally reduced reaction rates during the lowpressure periods helps to preserve the wave-rotor thermodynamic benefit.

An additional benefit of the slow-burn throughflow dual cycle is that it allows almost the full length of the channel to come in contact with fresh cold gas, resulting in a very favorable wall temperature profile. It may be possible to extend the dual cycle ided to multi cycles, where combustion of each charge continues for more than two cycles, if the exhaust expansion is weaker and a smaller fraction of combusted gas is exhausted per cycle. Throughput and pressure gain will be affected, and the optimal design will depend on the design wave-rotor temperature ratio.

It is evident from this discussion that there are many design possibilities for internal combustion wave rotor cycles. The development of the simulation code described here opens the door to further examination of such concepts.

\section{Acknowledgments}

This work was performed while the first author was a National Research Council-NASA Lewis Research Center Research Associate. 


\section{References}

Colella, P., Majda, A., and Roytburd, V., 1986. "Theoretical and Numerical Structure for Reacting Shock Waves," SIAM J. Sci. Stat. Comput., Vol. 7 , No. 4.

Heywood, J. B., 1988, Internal Combustion Engine Fundamentals, McGrawHill, New York

Larrouturou, B., 1991. "How to Preserve the Mass Fractions Positivity When Computing Compressible Multi-component Flows," J. Comput. Phys., Vol. 95, pp. $59-84$.

Magnussen, B. F., and Hjertager, B. H., 1976, “On Mathematical Modeling of Turbulent Combustion With Special Emphasis on Soot Formation and Combustion," I6th Symposium (International) on Combustion, Combustion Institute, pp. $719-729$.

Nalim, M. R., 1994, "Wave Cycle Design for Wave Rotor Engines With Limited Nitrogen Oxide Emissions," Ph.D. Thesis, Cornell University, Ithaca, NY.

Nalim, M. R., 1995, "Preliminary Assessment of Combustion Modes for Internal Combustion Wave Rotors," AlAA Paper No. 95-2801; NASA TM-107000.
Paxson, D. E., 1992, "A General Numerical Model for Wave Rotor Analysis," NASA TM- 105740

Paxson, D. E., 1995a, “A Comparison Between Numerically Modeled and Experimentally Measured Wave Rotor Loss Mechanisms," ALAA Journal of Propulsion and Power, Vol. i1. No. 5, pp. 908-914.

Paxson, D. E., 1995b, "A Numerical Model for Dynamic Wave Rotor Analysis." AIAA Paper No. 95-2800; NASA TM-106997.

Paxson, D. E., and Wilson, J., 1995. "Recent Improvements to and Validation of the One Dimensional NASA Wave Rotor Model,"' NASA TM-106913.

Roe, P. L., 1986, "Characteristic-Based Schemes for the Euler Equations," Ann. Review of Fluid Mech., Vol. 18, pp. 337-65.

Shreeve, R. P., and Mathur, A., eds., 1985, Proc. 1985 ONR/NAVAIR Wave Rotor Research and Technology Workshop, Naval Postgraduate School, Monterey, CA.

Welch, G. E., Jones, S. M., and Paxson, D. E., 1995, "Wave Rotor Enhanced Gas Turbine Engines," AIAA Paper No. 95-2799: NASA TM-106998, ARL-TR806.

You can now reach ASME Information Central representatives by e-mail. Simply

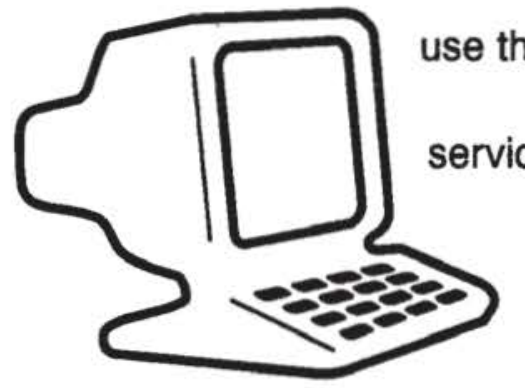

Information Central is easier than ever!

The American Society of Mechanical Engineers 